\title{
Sculpting the Stellar Cusp in the Galactic Center
}

\author{
Xian Chen ${ }^{1}$ \& Pau Amaro-Seoane ${ }^{1}$ \\ ${ }^{1}$ Max Planck Institut für Gravitationsphysik (Albert-Einstein-Institut), Am \\ Mühlenberg 1, D-14476 Potsdam, Germany.
}

\begin{abstract}
.
Observations of the innermost parsec surrounding Sgr A* - the supermassive black hole in the center of our Galaxy - have revealed a diversity of structures whose existence and characteristics apparently defy the fundamental principles of dynamics. In this article, we review the challenges to the dynamics theories that have been brought forth in the past two decades by the observations of the Galactic center (GC). We outline the theoretical framework that has been developed to reconcile the discrepancies between the theoretical predictions and the observational results. In particular, we highlight the role of the recently discovered sub-parsec stellar disk in determining the dynamics and resolving the inconsistencies. We also discuss the implications for the recent activity of Sgr A*.
\end{abstract}

Keywords: black holes, stellar dynamics, hydrodynamics, Galactic center, quasars

PACS numbers: $04.25 . \mathrm{Nx}, 98.10 .+\mathrm{z}$, 95.30.Lz, 98.35.Jk, 98.54.-h

\section{Introduction}

Since the late 1970s, there was already circumstantial evidence pointing to the existence of a supermassive black hole (SMBH) in the dynamical center of our Galaxy [1, 2. Later observations pinpointed the location of the $\mathrm{SMBH}$ at $\mathrm{Sgr} \mathrm{A}^{*}$, the compact radio object now defining the Galactic center $(\mathrm{GC})$ 3. The radiative characteristics of Sgr A* suggest that it is fueled by plasma at an extremely low rate $4,5,6$. These early discoveries are consonant with the hypothesis that SMBHs were born and growing in the early universe, manifesting themselves as active galactic nuclei (AGN) [7, 8], but today they are mostly "dead", lurking in the nuclei of quiescent galaxies [9, 10].

More solid evidence of the SMBH in the GC comes from stellar dynamics. In the past two decades, the advent of the adaptive optics and integral field spectroscopy allows us to detect stars in the close vicinity of Sgr $\mathrm{A}^{*}$ and monitor their orbits with high precision [11, 12, 13, 14, 15]). So far, dozens of B-type stars (so called "S-star cluster", or "S-cluster") have been discovered in a region as close as $<1$ " $\left(1^{\prime \prime} \simeq 0.04 \mathrm{pc} \simeq 0.13\right.$ light year at the GC) from Sgr $A^{*}[16,13,14,15$. Their nearKeplarian orbits have provided the hitherto most stringent constraint on the mass of a SMBH, which is $M_{\bullet} \simeq 4.3 \times 10^{6} M_{\odot}$ (e.g. [15]). Such a tight constraint firmly anchors our Milky Way (MW) toward the lower end of the correlation, shared among the galaxies in the local universe, between the masses of their central SMBHs and the properties (such as mass and stellar velocity dispersion) of their stellar components [17. 
Meanwhile, photometric and kinematic observations of the stars in a larger area surrounding Sgr A* have revealed a rich family of structures (see [18 for a review). Now it is clear that the S-cluster is the innermost part of a young star cluster, which is composed of mainly B-stars (each has a mass of $7-20 M_{\odot}$ and a typical age of $\sim 10-10^{2}$ Myrs) and extending continuously out to a distance of several pc from Sgr A* [19, 20, 21, 22, 23. This cluster spatially coincides with an old (several Gyr) population of red giants (RGs), whose surface density exhibits a characteristic "corelike" profile, such that at the innermost $0.5 \mathrm{pc}$ of the cluster, the surface density of the RGs becomes constant or possibly decreasing toward the central SMBH [20, 21, 24, 22. The young B-stars and the old RGs in the GC constitute the nuclear star cluster (NSC) of the MW. There is another dynamically distinct structure centered on Sgr $\mathrm{A}^{*}$ and spanning a radial range of $(0.04-0.5) \mathrm{pc}$, known as the "mini disk", which consists of $O\left(10^{2}\right)$ young $(6 \pm 2 \mathrm{Myr})$ and massive $\left(>20 M_{\odot}\right)$ Wolf-Rayet (WR) and O-type stars, in a configuration of a (possibly two) mildly thick disk(s). Apart from these discoveries, it is likely that so far we have spotted only the tip of the iceberg - the vast majority of the fainter, lower-mass stars may be "hiding" behind the bright glow in this very crowded field.

The rich phenomena unveiled in the GC offer a starting point for us to test our various hypotheses of the stellar and gaseous processes that may be responsible for the formation and evolution of SMBHs in general [25, 26]. However, when contrasting the observations with the theories, many inconsistencies occur [27]. For example, the core-like distribution of RGs around Sgr A* contradicts the longstanding hypothesis that stars in the potential well of a SMBH will relax due to two-body interactions to form a cusp whose stellar density should rise steeply toward the central SMBH (see [28, 29, 30, 31] for the original idea and 32] for a recent review). Furthermore, the existence of the S-cluster so close to Sgr A* imposes a paradox [33, 34]: the stars there cannot form in situ due to the violent environment, therefore they must be old so they have enough time to migrate in from outside due to dynamical friction, but S-stars are young. These discrepancies indicate that our comprehension of the stellar and gaseous processes close to a SMBH is far from complete.

During the past two decades, in the course of reconciling our dynamical model of the GC with the observations, new physical processes have been discovered and new theories have been formulated. These progresses have greatly enriched our understanding of the relationship between SMBHs and their close environments. In this contribution to the special issue, we will review the challenges we have been facing, as well as the potential solutions. In particular, we will highlight the recent discovery of the impact of the mini disk on the dynamics in the GC and its role in reconciling theories with observations.

The article is organized as follows. In section 2 we describe the "missing RG problem" and review the conventional solutions as well as their limits. We then introduce the most recent idea that it is caused by the repeated collisions of the RGs with the gaseous clumps in the fragmenting past of the mini disk. In Section 3, we outline the solutions to the "paradox of youth" imposed by the S-cluster. We also highlight the impact of the mini disk on the dynamical evolutions of the S-stars. The same dynamical effect of the disk offers a potential solution to the conundrum of "inversed mass segregation", that is manifested in the spatial distribution of young stars. This idea is elaborated in Section 4. Finally, in Section 5, we summarize, and generalize the conclusions to other quiescent galaxies. 


\section{Culprit for the Missing Red Giants}

\subsection{Is there a Bahcall-Wolf Cusp in the GC?}

The existence of a SMBH differentiates a region around it where the gravitational potential is dominated by that of the black hole $(\mathrm{BH})$. In the $\mathrm{GC}$, this region has a characteristic size of

$$
r_{h} \equiv \frac{G M_{\bullet}}{\sigma^{2}} \simeq 1.7 \mathrm{pc}\left(\frac{M_{\bullet}}{4 \times 10^{6} M_{\odot}}\right)\left(\frac{\sigma}{100 \mathrm{~km} \mathrm{~s}^{-1}}\right)^{-2},
$$

where $\sigma$ characterizes the velocity dispersion of the surrounding stars.

Inside the sphere of this "influence radius" $\left(r_{h}\right)$, a star revolves around the central SMBH on a near-Keplerian orbit, whose pericenter precesses in the orbital plane, at a rate determined by the gravitational potential of the NSC (Newtonian precession) as well as by the general-relativistic effect of the SMBH (GR precession). The star is unperturbed most of the time. But occasionally, it will encounter another star, exchanging energy and angular momentum with the interloper, which alters the orbits of both stars. Such "two-body interactions" redistribute energy and angular momentum between stars, a dynamical process called "relaxation". The equilibrium outcome, as has been predicted four decades ago, is a stellar cusp characterized by a steep-rising density profile toward the central SMBH, $\rho \propto r^{-7 / 4}$, also known as the "Bahcall-Wolf profile" 28, 29, 30, 31.

A number of studies more specific to the conditions in our Galaxy argued that a Bahcall-Wolf cusp is a robust outcome ([35, 36, 37, 38, 39, and see [40] for alternative). However, this prediction apparently contradicts the observational fact that the RGs in the GC, whose ages are several Gyr, do not show a cuspy distribution, but instead they exhibit a flat, core-like profile at $r<0.5$ pc [41, 20, 21, 24, 42]. This discrepancy is called "the problem of missing RGs". A number of solutions have been proposed. Most of them spring from two opposite standpoints: whether or not the RGs are reliable tracers of the underlying old stellar population.

2.1.1. Standpoint 1: No Bahcall-Wolf cusp in the GC If the RG core indeed reflects the intrinsic distribution of the old stars, then the density profile of the old stellar cusp is genuinely flat. From this point of view, one may imply that the Bahcall-Wolf cusp either was disrupted at some time within the past few Gyr or has never formed in the GC.

In fact, flat-cored galaxies are ubiquitous in the local universe [43, 17. A conventional explanation is that their NSCs are eroded due to the dynamical evolution of SMBH binaries, pairs of SMBHs that are repeatedly restored in galaxy centers following galaxy mergers or infalling of globular star clusters [44, 45, 46]. Such a galactic nucleus has the characteristic that the amount of (initially bound) stars that have been removed is correlated with the reduced mass of the SMBH binary [47, 48, 49]. Similar ideas have been applied to the GC 50, 51, 52, 53, 39. A contrast between a bona fide Bahcall-Wolf profile and the density profile in the GC shows that about an amount of $2 \times 10^{5} M_{\odot}$ of stars is currently missing 54 . The "mass deficit" could have been much larger in the past, because over a time span of several Gyr, stellar relaxation may have significantly reduced the core size by gradually recovering the Bahcall-Wolf profile 24. To remove this large amount of stars from the GC, the secondary BH is expected to be massive, probably $O\left(10^{5}\right) M_{\odot}$ [54]. A massive secondary $\mathrm{BH}$ could in principle induce perceivably large perturbations to the kinematics of $\operatorname{Sgr} \mathrm{A}^{*}$ and 
the surrounding stars, but so far there is no empirical evidence of these disturbances [55, 56, 57, 58, 59.

An alternative explanation to the non-existence of the Bahcall-Wolf cusp is that the cusp never has formed. This could be the case if the GC has been built up in a dissipationless fashion, for example, due to mergers of infalling star clusters [60, 61]. In this way, stars will be deposited preferentially at large distances from Sgr $\mathrm{A}^{*}$ where clusters become disrupted by the strong tidal field [62, and under two-body relaxation, it will take longer than a Hubble time to develop a BahcallWolf cusp. Recent numerical simulations demonstrated that consecutive mergers of about 10 massive star clusters indeed can build up a NSC with a flat density profile resembling that in the GC [63, 64, 65]. However, it is not clear whether the same model could reproduce as well the large degree of rotation which has been discovered in the central parsec of the NSC in our Galaxy [66, 67, 68,

2.1.2. Standpoint 2: A Bahcall-Wolf cusp in disguise On the other hand, one may consider the possibility that the RGs in the GC do not trace the underlying old stellar population. Then, it is possible that a Bahcall-Wolf cusp, composed of dim mainsequence stars and compact stellar objects (e.g. neutron stars and stellar-mass BHs), still exists in the GC. But a peculiar mechanism must be identified that can specifically deplete the RGs.

One such mechanism which has been proposed in the literature is stellar collision [41, 69]. It is more effective in depleting RGs than other types of stars because RGs have larger sizes, therefore larger collisional cross sections. Further calculations showed that stellar collision may explain the deficiency of RGs in the innermost $1^{\prime \prime}$ of the GC [70, given the condition that a Bahcall-Wolf cusp composed of compact stellar remnants does exit. But further out, because of the lower density of background stars, stellar collision becomes less frequent and more difficult to deplete the RGs there 71, 72.

\subsection{Hiding the Cusp during the Fragmenting Past of the Mini Disk}

Recently, we proposed a new mechanism [73, which does not rely on hypothetical ingredients, such as a secondary massive BH, a complicated merger history in the GC, or the pre-existence of a steep Bahcall-Wolf cusp. Meanwhile, the mechanism can deplete the RGs out to a large distance from Sgr $\mathrm{A}^{*}$ that matches observations. In this mechanism, the culprit for the missing RGs is the mini disk recently discovered in the GC (see Section 1). The key idea is that the WR/O stars, the current constituents of the disk, must have been born a few Myr ago out of massive (e.g. $10^{2} M_{\odot}$ ) gaseous clumps [74, 75, 76, and these clumps may destroy the envelopes of the RGs by repeatedly colliding with them. As a result, the RGs do not trace the distribution of the other types of stars.

At the core of the clumpy-disk mechanism is the process called "ram stripping". A RG ramming into a gaseous medium will gain certain amount of linear momentum in its outer envelope, and consequently, a fluid element in the outer envelope will receive a kick velocity. The kick velocity becomes comparable to the escape velocity at the surface of the RG if the collision happens, for example, in the central region of a quasar (the most luminous type of AGN), where the collision velocity increases to $>10^{3} \mathrm{~km} \mathrm{~s}^{-1}$ and the surface density of the gas medium, mostly likely in a disk configuration called "accretion disk", becomes as high as $10^{5} \mathrm{~g} \mathrm{~cm}^{-2}$. In such an 
extreme condition, each collision with the gas disk will result in a significant mass loss from the RG, and the entire RG envelope can be removed by about a dozen collisions 77.

The same condition for RG envelope depletion could be met in the GC. But first, some modifications to the conventional picture ([77) have to be made, because in the GC the accretion disk on average has a very low surface density, merely $\Sigma_{d} \sim 10^{4} M_{\odot} /(0.1 \mathrm{pc})^{2} \sim 200 \mathrm{~g} \mathrm{~cm}^{-2}$, which is inferred from the current stellar constituents of the mini disk. Instead, efficient ram stripping is caused by the collisions with the gas fragments (clumps), which must have existed in the past to give birth to the observed $O\left(10^{2}\right) \mathrm{WR} / \mathrm{O}$ stars in the disk. These clumps are so dense that the self-gravity is able to overcome the large turbulent pressure, allowing the clumps to collapse to form stars. Their surface densities are typically $10^{4-5} \mathrm{~g} \mathrm{~cm}^{-2}$, comparable to the surface densities of the quasar accretion disks. It has been shown that collisions with RGs hardly have any effects on such clumps 73 .

In the paper outlining the clumpy-disk mechanism [73, we considered 100 clumps $\left(10^{2} M_{\odot}\right.$ each) whose number and spatial distribution match the observations of the $\mathrm{WR} / \mathrm{O}$ stars in the mini disk. We have shown that each RG entering the sphere of radius of $0.1 \mathrm{pc}$ centered on $\mathrm{Sgr} \mathrm{A}^{*}$ will experience $2-60$ collisions with different clumps, therefore is prone to complete removal of its envelope. The exact number of collisions depends on the orbital inclination: a $\mathrm{RG}$ whose orbital plane is aligned with the mini disk is more frequently bombarded than a misaligned $R G$ would be.

The efficiency in depleting RGs by the clumpy-disk mechanism is determined by the following three elements. In particular, (i) and (ii) should both be effective. Otherwise, RG depletion will be restricted to a small region very close to Sgr $\mathrm{A}^{*}$, where the clumps are the densest, and consequently, the core will be smaller than what is observed in the GC.

(i) Non-linear mass loss: In the numerical simulations carried out by Armitage et al. [77, the collision is between a RG of a size of $150 R_{\odot}$ and an accretion disk of a surface density of $10^{4-5} \mathrm{~g} \mathrm{~cm}^{2}$. For these parameters, the collision will efficiently heat the RG, forcing the gas envelope of the RG to expand to a larger radius. Because of the expansion of the $R G$, it is suspected that during the next collision the envelope will lose twice more mass. Such a process of mass loss is non-linear. Assuming that the increment of mass loss during successive collisions is a factor of two, in other words, the non-linear factor is $f_{\text {loss }}=2$, then one will derive that after only 14 collisions the RG will be completely deprived of its envelope [73].

The degree of the non-linearity $\left(f_{\text {loss }}\right)$ is a crucial parameter of our model. It is determined by the relative efficiency of the heating (energy gain) and cooling processes in the RG, which in turn depends very much on the structure of the RG envelope. Since the most common RG has a radius smaller than $150 R_{\odot}$ and the energy gain is proportional to the projected area of the RG, it is expected that heating will be less effective for these RGs. On the other hand, for these typical RGs, cooling will be more important because of the shorter hydrodynamical timescale. As a result, in a more realistic situation, the non-linearity factor $f_{\text {loss }}$ may be close to unity, making the RGs more rigid against ram stripping. For example, if $f_{\text {loss }}=1.1$ (1.01), the number of collisions that is needed to completely remove the envelope of a RG increases to 80 (530). To quantify $f_{\text {loss }}$ for RGs of different sizes, more numerical simulations are needed. 
(ii) Longevity of the clumps: Unlike the clumps in conventional regions of massive star formation (SF), which are optically thin and will collapse on a free-fall timescale of $10^{3}$ yr [78, the clumps close to $\mathrm{Sgr} \mathrm{A}^{*}$ are extremely opaque due to their high densities 73. As a result, the heat released from gravitational collapse cannot escape freely, so the clumps are expected to contract slowly, on a timescale of several $10^{5} \mathrm{yr}$. The elongated lifetimes of the clumps near Sgr A* significantly increase the chance of RG-clump collisions.

The above rather unconventional picture of SF process may be further complicated if additional physical processes are taken into account, such as clump collision and gas accretion $([79,75,80,76])$. The clumps shaped by these additional processes are likely to have very different geometries as well as lifetimes. These differences may result in a variation of the probability of collision with the RGs, which deserves further investigation.

(iii) Number of clumps: The number of collisions mentioned above should be regarded as a lower limit, because in the calculation we neglected the contributions from the clumps with masses $10 M_{\odot}$ or smaller, whose number is observationally less well constrained. These lighter clumps in general have higher surface densities (see derivation in [73]), therefore will strip more mass from RG during each collision. Furthermore, the lighter clumps close to Sgr $\mathrm{A}^{*}$ normally have longer contraction times, because at birth their sizes are smaller so they radiatively dissipate the gravitational energy at a much slower rate 73 . The higher surface density and the longer lifetime compensate the smaller collisional cross section caused by the smaller size, making a light clump as effective as the more massive ones (e.g. $10^{2} M_{\odot}$ ) in terms of depleting the envelopes of RGs.

So far, it is unclear how many light clumps existed in the mini disk. Formation of a large amount of light clumps $\left(\sim 10 M_{\odot}\right.$ or less $)$ has been reported in the numerical simulations of the mini disk, especially those assuming effective gas cooling [75, 81, 82, 83, 84. But it is difficult to put the theoretical numbers into observational test, because the stellar descendants of the light clumps are normally faint, under the detection limit of the current telescopes [85, 22, 86]. There have been some hints, from the X-ray emission in the GC [87] and from the stellar dynamics of the mini disk [88, 89, suggesting that the total mass of the "unseen" stellar population is small. Deeper photometric and spectroscopic observations will help to better constrain the abundance of these clumps.

There are at least two possibilities to observationally test the clumpy-disk scenario for RG depletion. (1) Today, the cores released from the destructed RGs are still populating the GC, in the central $0.1 \mathrm{pc}$ region. Detection of them will strongly support the clumpy-disk scenario. These cores initially have high effective temperature (because of the thin envelopes) and are expected to be as luminous as their progenitor RGs [90]. Therefore, photometrically they resemble main-sequence stars of $3-4 M_{\odot}$, and deep spectroscopy is needed to reveal their real masses. (2) The surviving RGs, especially those most close to Sgr A* , should preferentially lie on the orbital planes that are inclined with respect to the mini disk. Otherwise, they cannot avoid large number of collisions with the clumps and their envelopes should have been depleted. 


\section{Randomizing S-stars}

\subsection{More than One Paradox}

Discovery of the S-cluster, and the observational follow-up in the past decade, has challenged the dynamical model of the GC with a series of dilemmas.

(i) Paradox of youth: It is generally believed that SF will be prohibited in the vicinity of Sgr $\mathrm{A}^{*}$, e.g. within a distance of $1^{\prime \prime} \simeq 0.04 \mathrm{pc}$, because of the violent environment, such as the strong tidal force, large turbulent velocity, and strong radiative background [33. Only old stars have had long enough time (several Gyr) to diffuse into the central region due to various stellar dynamical processes 32. This theoretical expectation, however, apparently contradicts the existence of the young (life expectancy of $6-200 \mathrm{Myr}$ ) S-cluster inside a region as close as $<1^{\prime \prime}$ from Sgr A*. This contradiction imposes a paradox which is termed "the paradox of youth" 33, 34.

This paradox can be (and has been) resolved because the S-stars can be produced further out and later brought in by some fast dynamical process (see 18 for a review). One such process is "binary separation". A star binary formed far away from Sgr A* could be scattered to a highly eccentric orbit, such that at the pericenter the binary will be tidally separated by the SMBH, leaving one star, which could be a B star, tightly bound to the SMBH and the other flying away with escape velocity [91, 92, 93, 94. This binary-separation model can explain the number and spatial distribution of the majority of the B stars within a distance of about 1 pc from Sgr A* 95, 96, 23]. The other possibility is "disk migration". A young star formed in the mini disk could lose its orbital energy and angular momentum by tidally interacting with the gas in the disk, then it will migrate toward the disk center [6]. In this model, a successful transportation of S-stars into the central 1 " region requires the existence of gas disk there, although currently there is no direct evidence supporting this prerequisite.

(ii) Paradox of randomness: Further analysis of the kinematics of the S-cluster revealed a second paradox. This is because in the binary-separation model, the captured stars initially have very high eccentricities, about $0.93-0.99$ (see the original work of 92 and 32 for a review), while in the disk-migration model, the stars in the disk normally have near-circular orbits 97 . Both eccentricity ranges are too narrow to match the observed "super-thermal" distribution $d N / d e \propto e^{2.6}$ of the S-stars [15], where $e$ is the orbital eccentricity.

This discrepancy has led to the necessity of including stellar-relaxation processes in the dynamical models, to randomize the angular momenta of S-stars, therefore broaden the initially narrow distribution in the eccentricity space. Two dynamical processes, which are inherent to any star-cluster system, have been considered first. One is the incoherent (random) scattering between two stars (two-body relaxation [98]), and the other is the coherent torquing between the stars on near-Keplerian orbits, known as (scalar) resonant relaxation, or (scalar) "RR" 99, 100. Numerical simulations including both processes showed that at least $20-25 \mathrm{Myr}$ is needed to recover the observed eccentricity distribution if S-stars are injected continuously through binary separations [101, 102, 103, 104, 105. If S-stars are produced by disk migration, a timescale longer than $100 \mathrm{Myr}$ will be needed, since the near-circular orbits preferentially have larger angular momenta 
103. These timescales, however, are longer than the life expectancy of one of the S-stars, S2/S0-2, which is about 6-10 Myr [34, 106, 107.

The situation becomes more intractable when one realizes that the relaxation timescales were derived under the assumption that a dense Bahcall-Wolf cusp exists in the GC, an assumption inconsistent with observations (Section 2). Without a Bahcall-Wolf cusp, the relaxation timescale is estimated to be much longer than $100 \mathrm{Myr}$ (e.g. [103]), then most S-stars will have difficulties evolving within their lifespans to their current orbital eccentricities. For this reason, the randomness of S-stars remains a problem.

(iii) Paradox of concurrence: Even if a Bahcall-Wolf cusp does exist so that paradox ii can be mitigated, there is yet another paradox. Occam's razor would favor a scenario that the young stars (B, O, and WR stars) in the GC have the same origin, e.g. in one single SF episode they were all produced. Three observations seem to support this scenario: (1) among the observed B stars the one with the shortest life expectancy $(6-10 \mathrm{Myr}$ for S2) seems to be coeval with the WR/O stars (6 \pm 2 Myrs old), (2) the luminosity function (probability distribution as a function of stellar luminosity) of the entire young stellar population is consistent with a single $\mathrm{SF}$ episode 2.5 -6 Myrs ago [86, and (3) recent analysis of the most complete data set no longer shows large discrepancy in the kinetic properties of the B and the WR/O stars [108.

Despite its simplicity, the coeval scenario conflicts with the theoretical prediction based on analysis of the relaxation timescales, that many B stars in the S-cluster should be older than 20-25 Myr. The root of this last conflict clearly is entangled with that of paradox ii. It also has difficulties explaining the apparent spatial segregation between $\mathrm{B}$ and $\mathrm{WR} / \mathrm{O}$ stars: not a single $\mathrm{WR} / \mathrm{O}$ star is inside the central $1^{\prime \prime}$ region but dozens of B stars are [27].

\subsection{A Rapidly Evolving Region Created by the Disk}

The above paradoxes highlight the incompleteness of the simplest model of the GC - it is still missing a mechanism that can efficiently randomize the angular momenta of the S-stars within $10 \mathrm{Myr}$. One possible solution is to introduce a massive perturber in the central $1^{\prime \prime}$ region of the GC. For example, the perturber could be an intermediate massive black hole $(\mathrm{IMBH})$ in the mass range of $(400 \sim 4,000) M_{\odot}$ and residing at a distance of about $0.01^{\prime \prime} \sim 1^{\prime \prime}$ from $\operatorname{Sgr} A^{*}$ [109, 58. However, it is observationally challenging to confirm the existence of such an IMBH so close to Sgr $\mathrm{A}^{*}$ [55, 56, 57, 59, 110].

Recently, we have put forward a new idea, based on analytical estimates of the timescales, that the perturber is the progenitor of the currently-observed mini disk [11]. An axisymmetric structure around a SMBH, such as the mini disk surrounding Sgr $\mathrm{A}^{*}$, will gravitationally torque the orbit of a star, forcing both its orbital eccentricity and its orbital orientation to change. The subsequent secular evolution is known as the "Kozai-Lidov" (KL) evolution ([112, 113, 114]), named after the two dynamists who in the 1960s pioneered the analysis of such a process in planetary systems. In the following, we will show that because of the existence of the mini disk, the S-stars in the GC are also subject to the KL evolution.

3.2.1. The Mini Disk in the Past There is a key difference between our model and those earlier ones studying the disk effect [115, 116, 117, 118, 23]. This difference has 
led to opposite conclusions on the effectiveness of the KL mechanism. The earlier models adopted a disk which is identical to the currently observed one: it has a total mass of $M_{d} \simeq 10^{4} M_{\odot}$, a surface-density distribution of $\Sigma_{d}(R) \propto R^{-\alpha}(\alpha=1.4 \sim 2)$, and most importantly, it extends from an outer radius of $R_{\text {out }} \simeq 12^{\prime \prime}(\simeq 0.5 \mathrm{pc})$ to an inner radius of $R_{\text {in }} \simeq 1^{\prime \prime}(\simeq 0.04 \mathrm{pc})$ [85. In this configuration, the KL evolution will actually be suppressed, because the "effective mass" of the disk that is causing the KL evolution is exceeded by the mass of the NSC that is enclosed by the orbit of a test star [18. Under such condition, the gravitational potential of the NSC can induce a substantial Newtonian precession to impair the coherence of the KL interaction.

However, there is no reason to assume that the properties of the mini disk have not evolved during the past several Myr. Since the SF efficiency is normally smaller than unity $[97,119,81,82,83,84$, it is reasonable to consider the possibility that initially the disk was more massive than $10^{4} M_{\odot}$, which is the total mass of the WR/O stars currently inside the disk [120, 85]. Furthermore, since the disk originally was composed of gas, which could shed angular momentum by viscosity, it was likely to be more extended toward the central SMBH $74,75,76,121$.

For these reasons, we adopt in our model $M_{d}=3 \times 10^{4} M_{\odot}$ and $R_{\text {in }}=10^{-6} \mathrm{pc}$ (innermost circular orbit) as the initial conditions of the disk, meanwhile we keep $R_{\text {out }}=0.5 \mathrm{pc}$ and $\Sigma_{d}(R) \propto R^{-1.4}$ ( $\alpha$ from [85]). In this new configuration, the central part of the disk within the radius of $R=1^{\prime \prime}$ contains a mass of $\Delta M_{d} \simeq 6,000 M_{\odot}$. This "inner disk" will play a crucial role in sustaining the KL evolutions of the S-stars. It is not surprising that today the inner disk is no longer present. This is because the gas would have been consumed by SF or accretion onto the SMBH [74, 75, 76, 122, 121]. After that, within several Myr, the stars, if there were any in the inner disk, would have been lifted out of the disk plane due to a particular consequence of RR, or "vectorial RR", which is to randomize the orientation of the stellar orbits [123, 124].

3.2.2. Numerical Simulations We have run numerical simulations to test the idea that the extended disk can induce KL evolutions of the S-stars. In these simulations, the gravitational potential is a combination of three components: (1) the extended disk (Section 3.2.1), (2) the central SMBH with a mass of $M_{\bullet}=4 \times 10^{6} M_{\odot}$, and (3) a spherical stellar cusp with shallow density profile $\rho \propto r^{-1.3}$ (Equation [4] from [18]), representing the observed NSC. The disk in our simulation is represented by 150 particles, each with a mass of $200 M_{\odot}$ and set on a circular orbit. This set up accounts for the clumpiness of the fragmenting disk. For the black hole, we adopt the pseudo-Newtonian potential [125. This potential allows us to investigate the impact of the GR precession on the KL evolution (e.g. [126, 127, 128]), which is expected to be important when $e$ becomes large. In our model, the mass of the NSC enclosed by a sphere of radius $1^{\prime \prime} \simeq 0.04 \mathrm{pc}$ is about $7,000 M_{\odot}$. This value is comparable to the mass of the "inner disk", which is $6,000 M_{\odot}$, as mentioned above.

Figure 1 shows the typical evolutions of the test stars with the same initial semimajor axis $a=0.02 \mathrm{pc}$, but different orbital eccentricities, i.e. $e=0.3$ and 0.9. Each curve is derived from a numerical integration of the equations of motion of a test particle. Initially, the orbital plane has a random orientation with respect to the disk plane. The right panel of Figure 1 shows that the evolution of the orbital eccentricity is mostly cyclic. This oscillatory behavior has been well studied, and can be attributed to the two integrals of motion of the system (see [129] for examples). The curves in the left panel of Figure1 1show more irregularities. This is partly because the Newtonian precession is more substantial for a test star with lower eccentricity - 

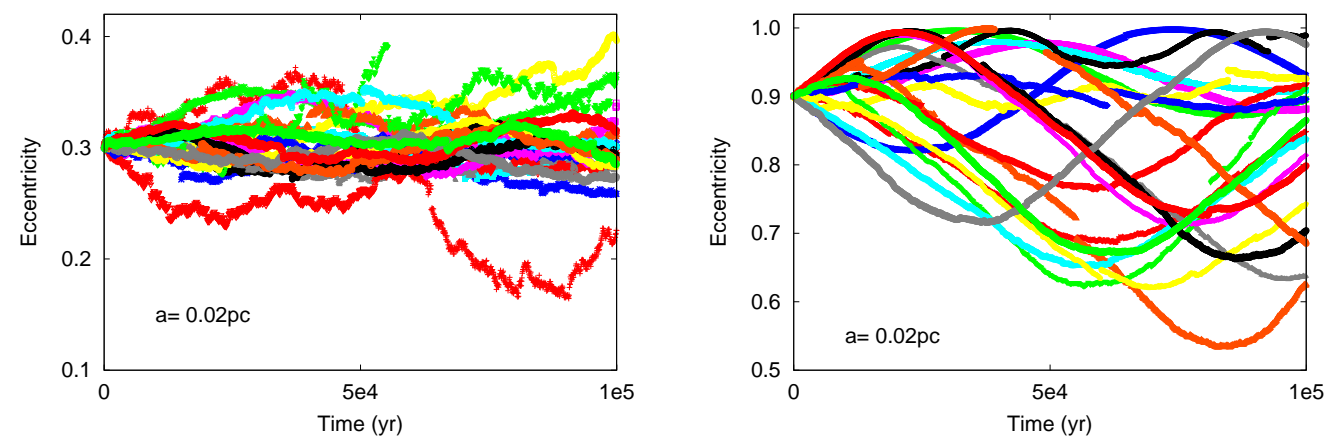

Figure 1. Examples of the Kozai-Lidov evolution excited by the extended disk. Test stars initially have the same semimajor axis, $a=0.02 \mathrm{pc}$. The initial orbital eccentricity is either $e_{0}=0.3$ (left panel), representing the S-stars formed in the disk-migration model, or $e_{0}=0.9$ (right panel), representing those in the binary-separation model. Different curves have different orbital inclinations (lines different colors) relative to the plane of the disk.

more mass from the NSC is enclosed by the orbit of the star. More importantly, it is due to the longer oscillation period - it takes longer time for the disk torque to alter the larger angular momentum (smaller $e$ ). To be more quantitative, the KL formalism (e.g. [129, 118, 114]) predicts that a test star will complete a KL cycle on a timescale of

$$
t_{K}(a, e) \sim l\left(M_{\bullet} / \Delta M_{d}\right) P(a),
$$

where $P(a)$ is the orbital period of the test star whose orbital semimajor axis is $a$, and $l=\sqrt{1-e^{2}}$ is the angular momentum of the test star normalized by the angular momentum of a circular orbit with the same $a$. The linear dependence on $l=\sqrt{1-e^{2}}$ stems from the fact that the disk torque is coherent during one KL cycle. In our model and given $a=0.02 \mathrm{pc}, t_{K}$ is about $3 \times 10^{5} \sqrt{1-e^{2}} \mathrm{yr}$. The results from our numerical simulations are consistent with this analytical prediction.

Figure 2 summaries more results from our numerical simulations. It is depicting the $(1-e)-a$ phase space of the stars at the innermost $10^{\prime \prime}$ region of the GC. In this diagram (see [11] for derivation), a star inside the region delimited by the two black lines is expected to be driven by the KL torque, therefore will evolve, mostly horizontally, on the KL timescale. The blue contours inside this region indicate the evolution timescales, defined by $|(1-e) / \dot{e}|$. We call this part of the phase space the "rapidly evolving region" (RER), because the evolution timescales are much shorter compared to the timescales outside, indicated by the gray contours. Outside the RER, the evolution timescales are determined by either two-body relaxation or RR, whichever is shorter. Here, the KL evolution will be quenched by either the Newtonian (the upper-right part of the phase space) or the GR precession (the lower-left part).

It is worth noting that our simulations, by construction, cannot capture the physical processes such as the two-body relaxation and (scalar) RR. But as can be seen in Figure 2, in the central 10" $\simeq 0.5 \mathrm{pc}$ of the Galaxy, both relaxation processes are operating on a timescale of $\sim\left(1-10^{3}\right)$ Myr (depending on $e$ ), much longer than the KL timescale. Therefore, on the timescale relevant to our simulations, which is shorter than $1 \mathrm{Myr}$, the effects of two-body relaxation and (scalar) RR are negligible. 
This justifies the omission of the two-body and resonant relaxation processes in our numerical simulations.

The gray boxes in Figure 2 mark the birth places of the S-stars predicted by the binary-separation and disk-migration models. Inside them, the red dots show the initial loci of the test stars in our simulations. Their initial semimajor axes are randomly sampled according to the observed density profile of $\rho \propto r^{-1.3}$ for the NSC 18. The other red dots outside the gray boxes represent the test stars which are selected to demonstrate the typical evolutions close to and outside the boundaries of the RER. For each test star, initially we assign a random orbital inclination, and then we numerically integrate the orbit for $3 \times 10^{5} \mathrm{yr}$. On a timescale longer than $3 \times 10^{5}$, the effect of vectorial RR is expected to be important (see below). Throughout the integration, we have recorded the minimum and maximum eccentricities of the orbit. In Figure2, the gray lines pointing away from the red dots indicate the loci of the tests stars when they obtain their extreme eccentricities. We note that in our simulations the $a$ of a test star is not conserved, because the gravitational potential is varying in time due to the lumpiness of the disk.

Figure 2 clearly shows that on a timescale of $3 \times 10^{5} \mathrm{yr}$, the stars inside the RER have moved prominently in the horizontal direction (in angular-momentum space), while those lying well outside do not move as much. This result confirms our idea that the KL evolution will be excited by the extended mini disk. Furthermore, the KL evolutions are more prominent for the stars formed in the binary-separation model (left gray box) than in the disk-migration one (right gray box). We have mentioned before that this difference is mainly due to the linear dependence of $t_{K}$ on $\sqrt{1-e^{2}}$.

On a timescale longer than $3 \times 10^{5}$, we expect the test stars in the RER to move further away from their initial locations. This further "mixing" in the angularmomentum space is driven by vectorial RR: it perturbs the orbital inclination $\theta$ of a test star 99 , and by changing the integrals of motion of the KL formalism which are functions of $\theta$ and $e$ 129, the perturbation will propagate into the amplitude of the KL oscillation. Such process is not captured by our scattering experiments, because vector $\mathrm{RR}$ is not implemented. According to the latest calculation [130, the timescale for vectorial RR to change $\theta$ is proportion to $P(a)$ and $\sqrt{1-e^{2}} /(1.05-0.3 e)$, and in our model with $\rho \propto r^{-1.3}$, it is shorter than $6 \mathrm{Myr}$ for a star at $a<1^{\prime \prime}$ (also see [111). This indicates that the KL mechanism, combined with the vectorial RR, could result in a rapid randomization of the S-stars inside the central 1" region of our Galaxy.

3.2.3. Further Test of the RER Scenario At this point, one could wonder what the distribution function $(d N / d e)$ would be like if S-stars are randomized by the RER. Some hints can be found in the analytical formulae of KL evolution. In the KL formalism, the time $\Delta t$ spent by a test star in a particular eccentricity interval $e \sim e+\Delta e$ is proportional to $e \Delta e / \sqrt{1+e^{2}}$ (e.g. [129]). The term $1 / \sqrt{1+e^{2}}$ is inversely proportional to the angular momentum of the star, and it stems from the very fact that an orbit with smaller angular momentum reacts more quickly to the KL torque. The additional factor $e$ ensures that the axisymmetric torque does not change $e$ of a star on circular orbit, because the two sections of the orbit on the opposite sides of the disk plane will receive equal amount of torque. Finally, we can derive $d N / d e=d t / d e=e / \sqrt{1+e^{2}}$, which follows from the fact that in a completely mixed system the number of stars $\Delta N$ in the interval $e \sim e+\Delta e$ is proportional to $\Delta t$. As has been shown in [111, the above distribution function is steeper than a thermal one, due to the additional term $1 / \sqrt{1+e^{2}}$, and it naturally and faithfully recovers the 


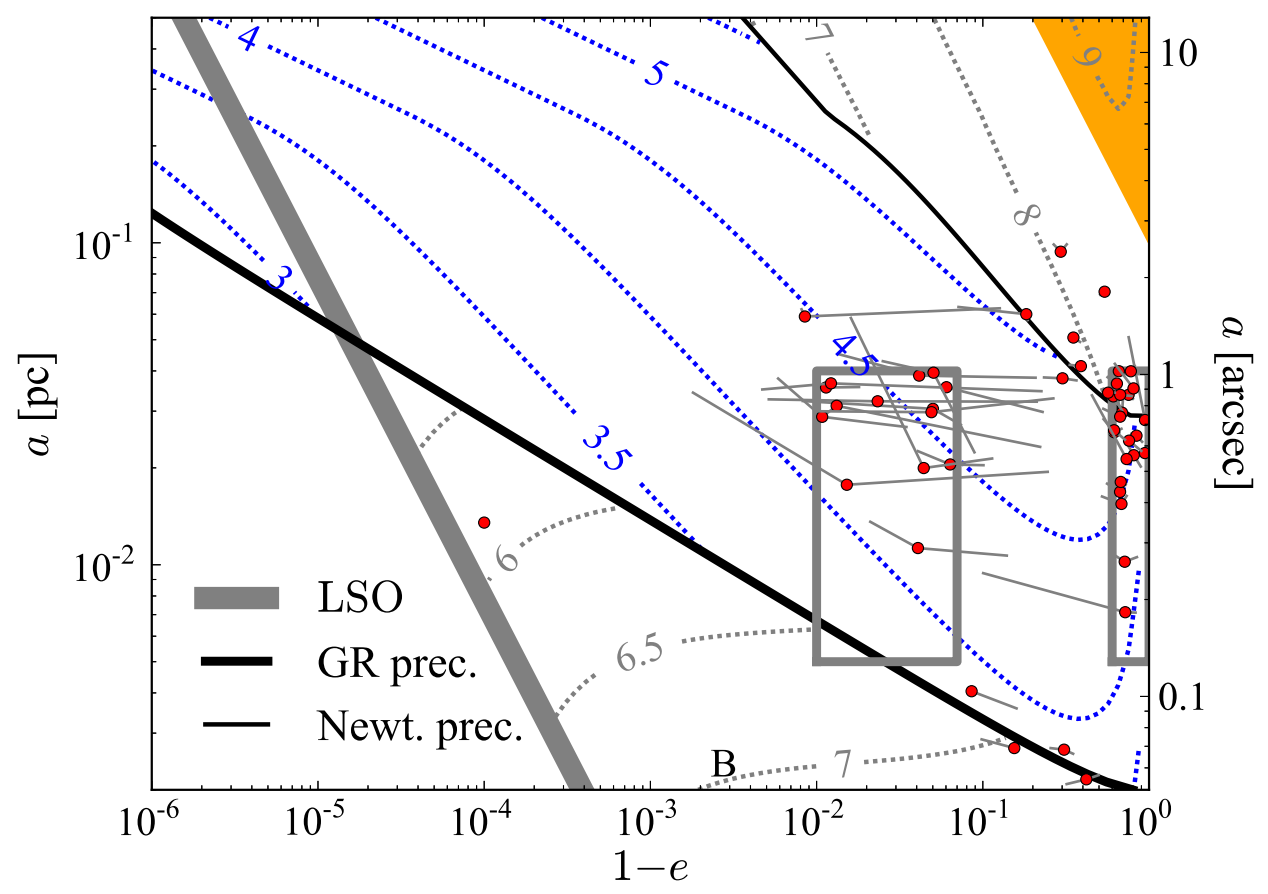

Figure 2. Evolutions of the test stars in the phase space of $1-e$ and $a$. The region delimited by the two black lines is the RER. In it, the evolution is dominated by the KL mechanism, and the blue dotted isochrones are associated with the logarithms of the KL timescales. Above the thin black line, the KL evolution will be quenched by Newtonian precession. Below the thick black line, the quenching is due to GR precession. In these two regions outside the RER, the evolution is dominated by either two-body relaxation or RR, and the gray dotted isochrones are associated with the logarithms of the two-body-relaxation or RR timescales, whichever is shorter. The thick gray line on the left-hand side corresponds to the last stable orbit (LSO) around Sgr A. The small orange triangles at the top right corner depicts the location of the red giants in the GC [24]. The two gray boxes depict the expected birth places of S-stars in the binary-separation and migration-in-disk models (also see [103]). The red dots mark the initial loci of the test stars in our simulations, and the gray lines show the extensions of the KL evolutions during $3 \times 10^{5} \mathrm{yr}$.

super-thermal distribution observed in the S-cluster.

The major caveat in the analytical approach is the assumption of a static disk. In fact, the timescale for a test star to "mix" with other stars in the RER is comparable to the vectorial $\mathrm{RR}$ timescale, the timescale on which the relevant part of the disk is evaporating. This last condition means that the disk torque is diminishing as the test star evolves. The efficiency of mixing in this situation deserves further investigation, and a self-consistent treatment of the time-dependent background may require numerical N-body simulations.

In the future if dimmer, older (than B-type) stars can be discovered in the GC, there would be an alternative, empirical approach of testing the RER scenario. Since old and young stars in the central $1^{\prime \prime}$ region respond indifferently to the disk torque, 
in principle they should obtain the same distribution function by now. Without the RER, however, the old stellar population may have had time to relax but not so much for the young stars (Section 3.1). Therefore, the orbital eccentricities of the old stars inside $1^{\prime \prime}$ contain crucial information about whether or not the RER exists.

3.2.4. Advantages of the RER Scenario One attractiveness of the RER scenario is that it offers a single, unified resolution to paradoxes ii and iii (Section 3.1).

- Before explaining how the RER scenario resolves the other paradoxes, it is worth emphasizing that it alone does not resolve paradox i; mechanisms such as binary separation and disk migration are needed to form the S-cluster in the first place.

- The RER shortens the relaxation timescale of the S-cluster to several Myr. Most importantly, the hypothetical Bahcall-Wolf cusp is no longer a prerequisite -a stellar background with shallow density profile, as has been observed in the GC, would suffice. This stellar distribution is fully compatible with the non-detection of a cuspy profile in the distribution of RGs.

- Because of RER, the B stars can be coeval with the WR/O stars. Earlier models without RER have difficulties conforming with this proposition: if all B stars formed simultaneously with WR/O stars about 6 Myr ago, two-body relaxation and $\mathrm{RR}$ will fail to recover the observed distribution of $e$.

So far, one puzzle remains. If B stars are coeval with $\mathrm{WR} / \mathrm{O}$ stars, what is the difference that causes the difference in the spatial distribution of these two types of stars? This question leads us to the following section.

\section{Depleting the Innermost WR/O Stars}

\subsection{An Inversed Mass Segregation}

In a close encounter between two stars, the principle of energy equipartition 98 predicts that the less massive star will eventually acquire a velocity that is greater than the velocity of the more massive star. The difference in velocity will lead to a spatial segregation of stars: the more massive population will sink toward the center of the potential well, meanwhile the lighter population will be expelled further out. However, the stellar distribution in the GC does not conform to this straightforward prediction. In the $\mathrm{GC}$, the $\mathrm{B}$ stars $\left(<20 M_{\odot}\right)$ are populating the innermost $1^{\prime \prime}$ region, constituting the S-cluster, but the more massive WR/O stars $\left(>20 M_{\odot}\right)$ lie exclusively further out. This phenomenon is known as the "inversed mass segregation" [27.

Further analysis of the kinematic data of the WR/O stars revealed several peculiar signatures, which have inspired some ideas of solving the inverse-mass-segregation problem. But so far none of the approaches is conclusive.

(i) Longterm monitoring of the stellar orbits revealed that roughly-speaking half of the GC WR/O stars are residing in one (possibly two) disk structure(s) 174, 120, 131, 132, 133. It is believed that these stars are the descendants of an accretion disk 74, 75. Today, the stellar disk truncates at a distance of about $0.8^{\prime \prime}$ from Sgr A* , and if extrapolated to a distance smaller than $0.8^{\prime \prime}$, there would be about 30 more $\mathrm{WR} / \mathrm{O}$ stars 85 . These $30 \mathrm{WR} / \mathrm{O}$ stars are not expected to remain in the disk plane, because during their ages of several Myr they will be lifted out of the disk plane by vectorial RR (see Section 3 for description). Therefore, the truncation of the stellar disk at a radius of $0.8^{\prime \prime}$ is not surprising. 
But the puzzle is that RR does not change the semimajor axes of stellar orbits, so the $30 \mathrm{WR} / \mathrm{O}$ stars, though no longer associated with the disk plane, should remain inside the central $1^{\prime \prime}$ region of our Galaxy.

To explain the fact that not a single WR/O star is observed in the central $1^{\prime \prime}$, it has been suggested that fragmentation, which is a necessary condition for SF, may be suppressed at the central part of the previously gaseous disk [75, 80, 76, 121. However, even though SF was indeed prohibited so that initially there was a hole in the mini disk, the hole would have been refilled of stars within a short timescale of several Myr due to the tidal interactions between the WR/O stars and the gas [76, 134, or due to the mutual interactions between the WR/O stars themselves [135, 136, 137. Because of these refilling mechanisms, it is difficult to understand the sharp inner edge in the spatial distribution of the WR/O stars.

(ii) The remaining half of the WR/O stars do not appear to associate with the disk structure(s) today, and their spatial distribution also exhibits an cutoff at about $1^{\prime \prime}$ from Sgr A [85, 138, 108. Such a distribution has been interpreted in the context of the binary-separation scenario 95, 96, 27. In this scenario, in-situ SF is prohibited in the central $1^{\prime \prime}$ region around $\mathrm{Sgr} \mathrm{A}^{*}$, and the stars there were produced solely by the binary-separation mechanism (Section 3.11). Numerical calculations showed that the binaries supplied by this mechanism are mostly from a distance of about $5 \mathrm{pc}$ from $\mathrm{Sgr} \mathrm{A}^{*}$. This radial range coincides with the region where the abundance of $\mathrm{WR} / \mathrm{O}$ binaries appears to be negligible.

The matter recently becomes more uncertain. On one hand, as mentioned above, theoretical studies on the dynamics of the mini disk predict a substantial feeding rate of the disk stars to the vicinity of Sgr A* 116, 117, 135, 136, 137. On the other, more WR/O binaries have been discovered in the mini disk 139, 140, 141, 142. These new discoveries point to a possibly non-negligible supply rate of WR/O stars into the central 1" region around Sgr A*, which revives the problem of inversed mass segregation.

\subsection{Depleting the WR/O Stars in the RER}

Now we know that it is difficult to completely prevent WR/O stars from forming in the central $1^{\prime \prime}$ of the Galaxy. Therefore, it is logical to seek a mechanism that can efficiently deplete them. In [111, we have pointed out that the RER can fulfill the task of preferentially depleting the WR/O stars in the designated region, by driving them to highly eccentric orbits so that at the orbital pericenters they will be tidally disrupted by the central SMBH. In this section, we describe in more detail how the RER mechanism would work.

4.2.1. The RER Mechanism A star wandering too close to Sgr A* will be tidally disrupted by the SMBH. The critical distance is approximately

$$
r_{t} \simeq r_{*}\left(\frac{M_{\bullet}}{m_{*}}\right)^{1 / 3} \simeq 4 \times 10^{-6} \mathrm{pc}\left(\frac{r_{*}}{R_{\odot}}\right)\left(\frac{m_{*}}{M_{\odot}}\right)^{-1 / 3},
$$

where $m_{*}$ and $r_{*}$ denote the mass and the radius of the star. This characteristic radius, $r_{t}$, known as the "tidal radius" [143, 144, sets a critical eccentricity $e_{t}=1-r_{t} / a$ for the orbit of a star, such that the star must have $e<e_{t}$ to avoid tidal disruption. The linear dependence of $r_{t}$ on $r_{*}$ hints that stars of larger sizes are more susceptible to tidal disruption. At first glance, $r_{t}$ is much smaller than the orbital semimajor axes of 
the S-stars, which may give the impression that tidal stellar disruption is rare. This is not true, as we will show below, when the RER is taken into account.

The function $e_{t}(a)$ imposes a new type of boundary in the phase space of $(1-e)$ and $a$, beyond which stars cannot exist. The two boundaries corresponding to the tidal radii of $\mathrm{WR}$ and $\mathrm{O}$ stars are illustrated in Figure 3 as the dashed lines. We adopt $\left(m_{*}, r_{*}\right)=\left(60 M_{\odot}, 80 R_{\odot}\right)$ for the WR stars and $\left(m_{*}, r_{*}\right)=\left(25 M_{\odot}, 60 R_{\odot}\right)$ for the $\mathrm{O}$ stars. The other model parameters are the same as in Figure 2. The stellar radii adopted here are somewhat $3-4$ times larger than the values for the main-sequence stars of the same masses. We choose the larger values because the $\mathrm{WR} / \mathrm{O}$ stars in the GC are known to have evolved off the main sequence and entered a transitional super-giant phase [120, 85].

Figure 3 shows that several Myr ago when the mini disk was more massive and extended, the RER intersected with the tidal-disruption boundaries at about $a>0.15^{\prime \prime} \simeq 0.006 \mathrm{pc}$. Because of this, no WR/O star can survive in the cyan-shaded region where $0.15^{\prime \prime}<a<0.8^{\prime \prime}$ : a WR/O star born in it, whatever $e$ it initially may have, will be transported horizontally by the RER to the location of $e_{t}$, and get tidally disrupted. The timescale for the transportation, as discussed in Section 3.2 .2 as well as in [11], is determined by vectorial RR, and is shorter than 6 Myrs for a star at $a<1^{\prime \prime}$. This range of timescale is comparable or shorter than the ages of the WR/O stars in the GC, therefore it is consistent with the scenario that the innermost WR/O stars were depleted due to the existence of the RER.

Figure 3 also indicates that WR/O stars may survive at $a>0.8^{\prime \prime}$, to the right of the upper boundary of the RER, where the evolution timescales are typically longer than $10 \mathrm{Myr}$. In fact, those WR/O stars which are feasible for both spectroscopic identification and 3-D position/velocity measurement are all detected there (red dots in Figure 3). In principle, a WR/O star may also survive at $a<0.15^{\prime \prime}$, in the region delimited by the lower boundary of the RER (thick solid line) and the tidal-disruption radius (dashed line), because there the evolution timescales are also long. However, we have not detected WR/O stars there, maybe because no WR/O was born in that small region: an extrapolation of the current spatial distribution of $\mathrm{WR} / \mathrm{O}$ stars would result in $<1$ star residing at $a<0.15^{\prime \prime}$ 85.

Now we proceed to explain why most B stars can be retained in the central $1^{\prime \prime}$ region surrounding Sgr $\mathrm{A}^{*}$. It can be understood by looking at Figure 4 , where the cyan-shaded area depicts the radial range in the phase space where a B star will eventually be tidally disrupted. This tidal-disruption region for B stars is much narrower in the radial range than that of the $\mathrm{WR} / \mathrm{O}$ stars. This is because a typical main-sequence B-star of mass $m_{*}=7 M_{\odot}$ has a much smaller radius, $r_{*} \simeq 4 R_{\odot}$, than the radius of an evolved $\mathrm{WR} / \mathrm{O}$ star, therefore it has a much smaller $r_{t}$ too. This predicted gap for B stars matches remarkably well the current distribution of the S-stars (blue dots in the right panel of Figure 4), supporting the idea that the RER does play a role in depleting the stars in the GC. For stars less massive than $7 M_{\odot}$, tidal disruption is expected to be less important, because these stars have an even smaller $r_{t}$, so the radial range relevant to tidal disruption becomes diminishing. It will be interesting to observationally test this last prediction.

4.2.2. Constraining the Initial Mass of the Disk The condition of retaining the Sstars in the central $1^{\prime \prime}$ region around Sgr A* in fact puts an upper limit on the mass of the mini disk. For example, if we had adopted in our model a larger mass for the initial disk, i.e. $M_{d}>3 \times 10^{4} M_{\odot}$, the RER in the $(1-e)-a$ diagram would become 


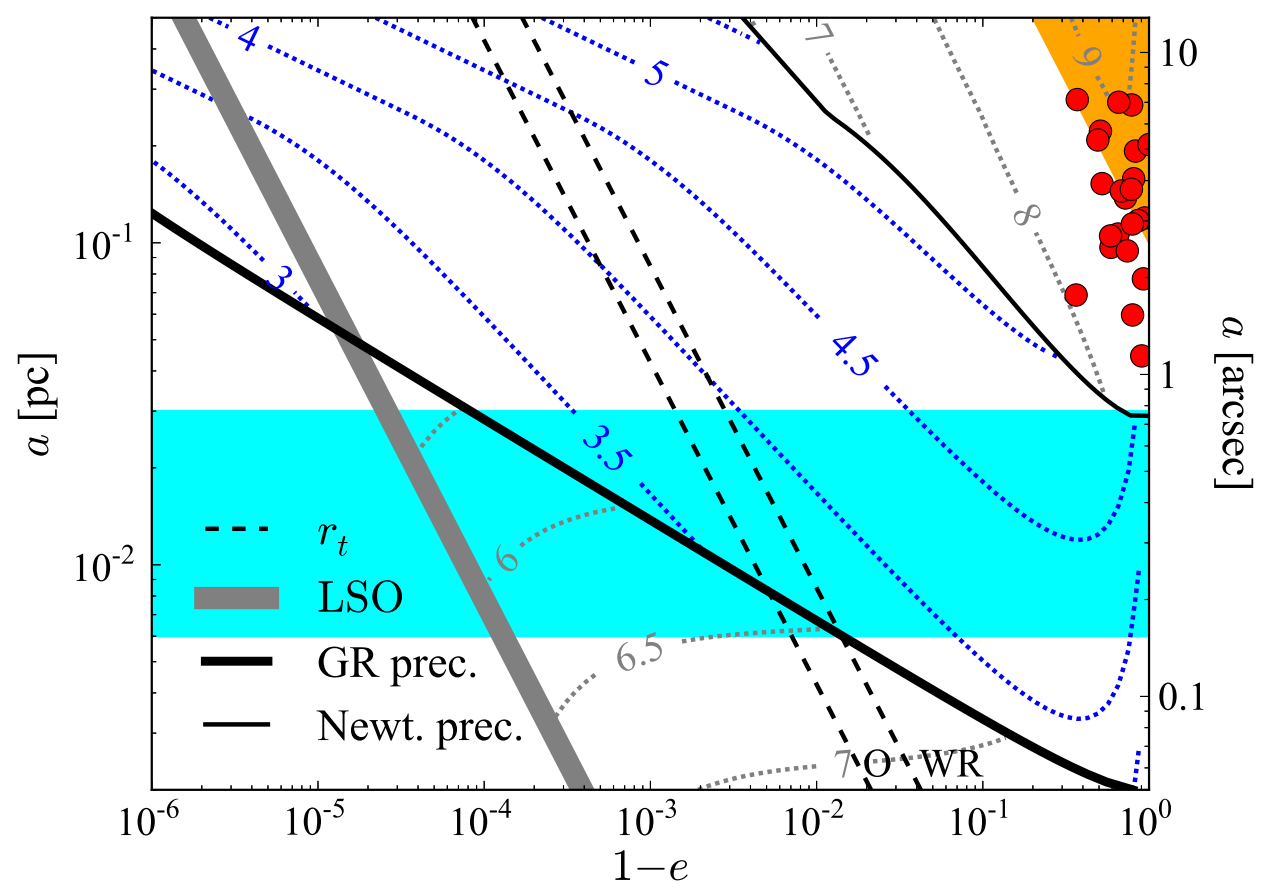

Figure 3. Radial range in the phase space where WR/O stars will be tidally disrupted by Sgr A* (cyan-shaded area). The dashed lines labeled with "O" and "WR" correspond to the tidal radii for, respectively, $\mathrm{O}$ and WR stars. The red dots represent the subsample of WR/O stars from [120] which are spectroscopically identified and have $3-\mathrm{D}$ position/velocity measurements. The rest lines have the same meaning as those in Figure 2
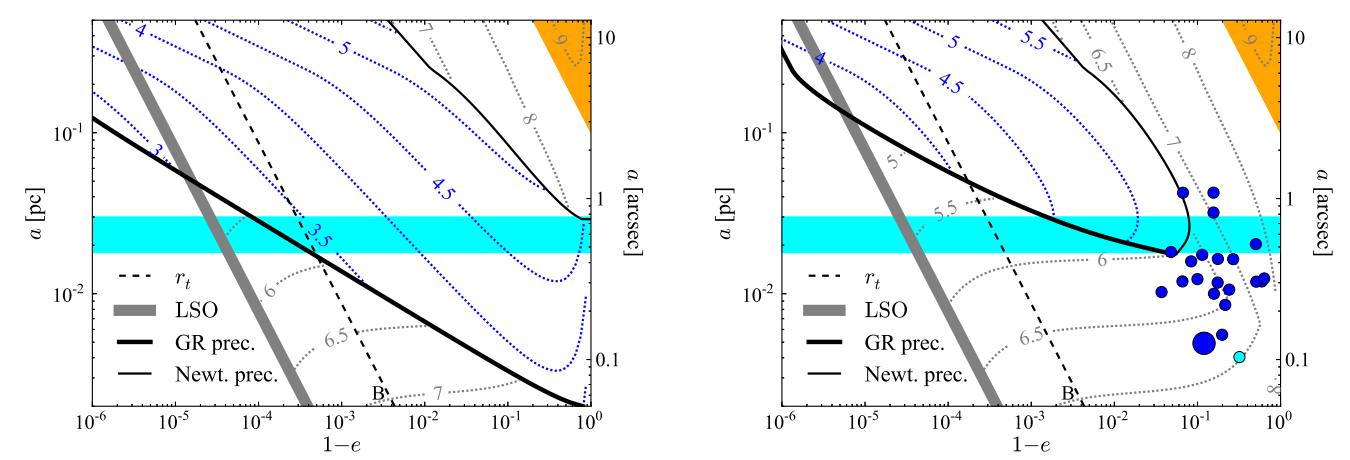

Figure 4. Left: The same as Figure 3 but for tidal disruption of B stars (assuming $m_{*}=7 M_{\odot}$ and $r_{*}=4 R_{\odot}$ ). Right: The same as the left panel, but calculated with the parameters of the present structures in the GC today, with a disk of mass $10^{4} M_{\odot}$ and inner radius at $1^{\prime \prime} \simeq 0.04 \mathrm{pc}$. The dots represent the S-stars not associated with the mini disk (small-blue 15]), S2/S0-2 (big-blue, the brightest S-star 34, 106, and S102/S0-102 (small-cyan [145), the S-star with the shortest period known. 
wider both vertically and horizontally. This change would result in a broader gap in the radial distribution of the S-stars, which would readily contradict the observations. For this reason, in [111] we have constrained the initial disk mass to be less than $3 \times 10^{4} M_{\odot}$.

One uncertainty, which prevents us from pinning down the exact mass of the disk, is the initial surface density profile of the disk. We now study the consequence of this uncertainty. In the previous calculations, we assumed that the disk has a power-law density profile, $\Sigma_{d} \propto R^{-\alpha}$, where $\alpha=1.4$, which is derived from the present distribution of the $\mathrm{WR} / \mathrm{O}$ stars 85 . So if the total mass of the disk is $M_{d}(R<0.5 \mathrm{pc})=3 \times 10^{4} M_{\odot}$, there will be an amount of $6,000 M_{\odot}$ of mass inside the radius of $1^{\prime \prime}$ of the disk (insensitive to $R_{\text {in }}$ as long as $R_{\text {in }} \ll 1^{\prime \prime}$ ). This amount of mass is comparable to the total mass of the stars residing at the innermost $1^{\prime \prime}$ of the NSC, therefore Newtonian precession does not impair the KL evolution [118. and the RER emerges.

For an arbitrary $\alpha$, the condition of sustaining a RER in the central $1^{\prime \prime}$ of the GC meanwhile retaining most of the B stars in the same region is, roughly speaking, that the mass in the central $1^{\prime \prime}$ of the disk $\left[M_{d}(R<0.04 \mathrm{pc})\right]$ should be comparable to and not significantly exceed that in the NSC $\left[M_{c}(R<0.04 \mathrm{pc}) \simeq 7,000 M_{\odot}\right]$. This condition, $M_{d}(R<0.04 \mathrm{pc}) \simeq M_{c}(R<0.04 \mathrm{pc})$, leads to an estimate on the total mass of the mini disk,

$$
\begin{aligned}
& M_{d}(R<0.5 \mathrm{pc}) \simeq(50 / 4)^{2-\alpha} M_{c}(R<0.04 \mathrm{pc}) \quad \text { if } \alpha<2, \text { and } \\
& M_{d}(R<0.5 \mathrm{pc}) \simeq[\ln (50) / \ln (4)] M_{c}(R<0.04 \mathrm{pc}) \quad \text { if } \alpha=2,
\end{aligned}
$$

which depends only on two observables, $\alpha$ and $M_{c}(R<0.04 \mathrm{pc})$. Here, we do not consider $\alpha>2$, because the total disk mass is divergent in such an initial condition. While $M_{c}(R<0.04 \mathrm{pc})$ is estimated to be about 7,000 $M_{\odot}$ [18, the value of $\alpha$ is less certain (see [137] and references therein). In our fiducial case with $\alpha=1.4$, we find $M_{d}(R<0.5 \mathrm{pc}) \simeq 3 \times 10^{4} M_{\odot}$. Otherwise, if $\alpha=1$ or 2 [137, we find $M_{d}(R<0.5 \mathrm{pc})$ is approximately $9 \times 10^{4} M_{\odot}$ or $2 \times 10^{4} M_{\odot}$. These numbers indicate that initially the mass of the mini disk is a few percent, or less, of the mass of the central SMBH.

\section{Summary and Outlook}

Decades of observations of the GC have revealed three puzzling phenomena, i.e. the problem of missing RGs, the paradox of youth, and the inversed mass segregation. They apparently defy the fundamental astrophysical principles. Explanations of these phenomena have been sought separately, and they often resort to hypothetical structures that so far have not been detected in the GC. As a result, the dynamical model of the GC progressively becomes more complicated.

In this article, we have identified a single, unified resolution to all the three conundrums, by adding only one ingredient which has been missing in the previous models -the dynamical effects of the recently discovered mini disk. We have shown that the clumps formed during the fragmenting past of the disk could efficiently strip the envelopes off the RGs in the central $0.1 \mathrm{pc}$ of the NSC, therefore duplicate the observed flat spatial distribution of the RGs. Considering that the disk probably was more massive and also had extended to a smaller radius in the past, we show that it would impose a RER in the central 1" $(0.04 \mathrm{pc})$ region around Sgr A*, in which the S-stars would be driven to cyclic evolutions (KL evolution) in the eccentricities space. 
These induced KL evolutions could quickly randomize the angular momenta of the S-stars, therefore can resolve the paradox of youth. The discovery of the RER also solves the problem of the inversed mass segregation, because the WR and O-stars in the central $1^{\prime \prime}$ region will intermittently attain ample eccentricities that will lead to their tidal disruptions by Sgr A* .

Our results point to the picture that several Myr ago our GC was an AGN. This picture has been repeatedly invoked to explain an increasing number of observations 146, 147, 148, 149, 150, 151, 152, 153. The results presented in this article provide an additional evidence, from a different, dynamics point of view. Further more, our study indicates that the AGN was powered by gas accretion as well as by tidal disruptions of WR/O stars.

We also find an upper limit on the total mass of the previously gaseous disk, which should be several percent of the mass of the central SMBH. Existence of such a small disk is consistent with the contemporary scenario that "small" SMBHs with $M_{\bullet}<10^{7} M_{\odot}$ are mostly powered by stochastic gas inflow (e.g. [154, 155]). In the absence of a preferential direction for gas supply, the above incoherent mode of mass accumulation is likely to spin down the central SMBH [156, 157, 158, 159, a prediction that can be tested by direct measurements of the spin of Sgr A* (e.g. using the event horizon telescopeit). However, it is likely that future observations and theoretical investigations of the $\mathrm{GC}$ will continue to surprise and enlighten us.

\section{Acknowledgment}

This work has been supported by the Transregio 7 "Gravitational Wave Astronomy" financed by the Deutsche Forschungsgemeinschaft DFG (German Research Foundation).

\section{References}

[1] E. R. Wollman, T. R. Geballe, J. H. Lacy, C. H. Townes, and D. M. Rank. NE II 12.8 micron emission from the galactic center. II. Astrophys. J. Lett., 218:L103-L107, December 1977.

[2] J. H. Lacy, C. H. Townes, T. R. Geballe, and D. J. Hollenbach. Observations of the motion and distribution of the ionized gas in the central parsec of the Galaxy. II. Astrophys. J., 241:132-146, October 1980.

[3] K. Y. Lo. The galactic center compact nonthermal radio source. In D. C. Backer, editor, The Galactic Center, volume 155 of American Institute of Physics Conference Series, pages 30-38, April 1987.

[4] R. Narayan, I. Yi, and R. Mahadevan. Explaining the spectrum of Sagittarius A* with a model of an accreting black hole. Nature, 374:623-625, April 1995.

[5] R. Narayan, R. Mahadevan, J. E. Grindlay, R. G. Popham, and C. Gammie. Advectiondominated accretion model of Sagittarius $\mathrm{A}^{*}$ : evidence for a black hole at the Galactic center. Astrophys. J., 492:554-568, January 1998.

[6] F. Yuan, S. Markoff, and H. Falcke. A Jet-ADAF model for Sgr A*. Astron. Es Astrophys., 383:854-863, March 2002.

[7] M. Schmidt. 3C 273 : A Star-Like Object with Large Red-Shift. Nature, 197:1040, March 1963.

[8] D. Lynden-Bell. Galactic Nuclei as Collapsed Old Quasars. Nature, 223:690-694, August 1969.

[9] A. Soltan. Masses of quasars. Mon. Not. Roy. Astron. Soc. , 200:115-122, July 1982.

[10] Q. Yu and S. Tremaine. Observational constraints on growth of massive black holes. Mon. Not. Roy. Astron. Soc. , 335:965-976, October 2002.

[11] A. M. Ghez, M. Morris, E. E. Becklin, A. Tanner, and T. Kremenek. The accelerations of stars orbiting the Milky Way's central black hole. Nature, 407:349-351, September 2000.

$\ddagger$ http://www.eventhorizontelescope.org 
[12] A. Eckart, R. Genzel, T. Ott, and R. Schödel. Stellar orbits near Sagittarius A*. Mon. Not. Roy. Astron. Soc. , 331:917-934, April 2002.

[13] F. Eisenhauer, R. Genzel, T. Alexander, R. Abuter, T. Paumard, T. Ott, A. Gilbert, S. Gillessen, M. Horrobin, S. Trippe, H. Bonnet, C. Dumas, N. Hubin, A. Kaufer, M. KisslerPatig, G. Monnet, S. Ströbele, T. Szeifert, A. Eckart, R. Schödel, and S. Zucker. SINFONI in the Galactic Center: Young Stars and Infrared Flares in the Central Light-Month. Astrophys. J., 628:246-259, July 2005.

[14] A. M. Ghez, S. Salim, S. D. Hornstein, A. Tanner, J. R. Lu, M. Morris, E. E. Becklin, and G. Duchêne. Stellar Orbits around the Galactic Center Black Hole. Astrophys. J., 620:744757, February 2005

[15] S. Gillessen, F. Eisenhauer, S. Trippe, T. Alexander, R. Genzel, F. Martins, and T. Ott. Monitoring Stellar Orbits Around the Massive Black Hole in the Galactic Center. Astrophys. J., 692:1075-1109, February 2009.

[16] R. Schödel, R. Genzel, T. Ott, and A. Eckart. The Galactic Center stellar cluster: The central arcsecond. Astronomische Nachrichten Supplement, 324:535-541, September 2003.

[17] J. Kormendy and L. C. Ho. Coevolution (Or Not) of Supermassive Black Holes and Host Galaxies. Annu. Rev. Astron. Astrophys., 51:511-653, August 2013.

[18] R. Genzel, F. Eisenhauer, and S. Gillessen. The Galactic Center massive black hole and nuclear star cluster. Reviews of Modern Physics, 82:3121-3195, October 2010.

[19] R. Schödel, A. Eckart, T. Alexander, D. Merritt, R. Genzel, A. Sternberg, L. Meyer, F. Kul, J. Moultaka, T. Ott, and C. Straubmeier. The structure of the nuclear stellar cluster of the Milky Way. Astron. \& Astrophys., 469:125-146, July 2007.

[20] R. M. Buchholz, R. Schödel, and A. Eckart. Composition of the galactic center star cluster. Population analysis from adaptive optics narrow band spectral energy distributions. Astron. ES Astrophys., 499:483-501, May 2009.

[21] T. Do, A. M. Ghez, M. R. Morris, J. R. Lu, K. Matthews, S. Yelda, and J. Larkin. High Angular Resolution Integral-Field Spectroscopy of the Galaxy's Nuclear Cluster: A Missing Stellar Cusp? Astrophys. J., 703:1323-1337, October 2009.

[22] T. Do, J. R. Lu, A. M. Ghez, M. R. Morris, S. Yelda, G. D. Martinez, S. A. Wright, and K. Matthews. Stellar Populations in the Central 0.5 pc of the Galaxy. I. A New Method for Constructing Luminosity Functions and Surface-density Profiles. Astrophys. J., 764:154, February 2013

[23] A.-M. Madigan, O. Pfuhl, Y. Levin, S. Gillessen, R. Genzel, and H. B. Perets. On the Origin of the B-stars in the Galactic Center. Astrophys. J., 784:23, March 2014.

[24] D. Merritt. The Distribution of Stars and Stellar Remnants at the Galactic Center. Astrophys. J., 718:739-761, August 2010.

[25] F. Melia and H. Falcke. The Supermassive Black Hole at the Galactic Center. Annu. Rev. Astron. Astrophys., 39:309-352, 2001.

[26] T. Alexander. Stellar processes near the massive black hole in the Galactic center [review article]. Phys. Rep., 419:65-142, November 2005.

[27] T. Alexander. Key Questions about Galactic Center Dynamics. In M. R. Morris, Q. D. Wang, and F. Yuan, editors, The Galactic Center: a Window to the Nuclear Environment of Disk Galaxies, volume 439 of Astronomical Society of the Pacific Conference Series, page 129, May 2011.

[28] P. J. E. Peebles. Star Distribution Near a Collapsed Object. Astrophys. J., 178:371-376, December 1972. Original paper on the stellar distribution around massive black hole.

[29] J. N. Bahcall and R. A. Wolf. Star distribution around a massive black hole in a globular cluster. Astrophys. J., 209:214-232, October 1976. take anisotropy into account.

[30] S. L. Shapiro and A. P. Lightman. The distribution of stars around a massive black hole. Nature, 262:743-745, August 1976. Analytical arguments for the scaling relations.

[31] A. P. Lightman and S. L. Shapiro. The distribution and consumption rate of stars around a massive, collapsed object. Astrophys. J., 211:244-262, January 1977. First modeling using the FP formalism.

[32] P. Amaro-Seoane. Stellar dynamics and extreme-mass ratio inspirals. ArXiv e-prints, May 2012. steady-state distribution, flux independent of energy.

[33] M. Morris. Massive star formation near the Galactic center and the fate of the stellar remnants. Astrophys. J., 408:496-506, May 1993.

[34] A. M. Ghez, G. Duchêne, K. Matthews, S. D. Hornstein, A. Tanner, J. Larkin, M. Morris, E. E. Becklin, S. Salim, T. Kremenek, D. Thompson, B. T. Soifer, G. Neugebauer, and I. McLean. The First Measurement of Spectral Lines in a Short-Period Star Bound to the Galaxy's Central Black Hole: A Paradox of Youth. Astrophys. J. Lett., 586:L127-L131, April 2003. 
[35] M. Freitag, P. Amaro-Seoane, and V. Kalogera. Stellar Remnants in Galactic Nuclei: Mass Segregation. Astrophys. J., 649:91-117, September 2006.

[36] C. Hopman and T. Alexander. The Effect of Mass Segregation on Gravitational Wave Sources near Massive Black Holes. Astrophys. J. Lett., 645:L133-L136, July 2006.

[37] M. Preto and P. Amaro-Seoane. On Strong Mass Segregation Around a Massive Black Hole: Implications for Lower-Frequency Gravitational-Wave Astrophysics. Astrophys. J. Lett., 708:L42-L46, January 2010.

[38] P. Amaro-Seoane and M. Preto. The impact of realistic models of mass segregation on the event rate of extreme-mass ratio inspirals and cusp re-growth. Classical and Quantum Gravity, 28(9):094017, May 2011.

[39] A. Gualandris and D. Merritt. Long-term Evolution of Massive Black Hole Binaries. IV. Mergers of Galaxies with Collisionally Relaxed Nuclei. Astrophys. J., 744:74, January 2012.

[40] F. Antonini and D. Merritt. Dynamical Friction around Supermassive Black Holes. Astrophys. J., 745:83, January 2012.

[41] R. Genzel, N. Thatte, A. Krabbe, H. Kroker, and L. E. Tacconi-Garman. The Dark Mass Concentration in the Central Parsec of the Milky Way. Astrophys. J., 472:153, November 1996.

[42] F. Yusef-Zadeh, H. Bushouse, and M. Wardle. Hubble Space Telescope Observations of the Stellar Distribution near Sgr A*. Astrophys. J., 744:24, January 2012.

[43] S. M. Faber, S. Tremaine, E. A. Ajhar, Y.-I. Byun, A. Dressler, K. Gebhardt, C. Grillmair, J. Kormendy, T. R. Lauer, and D. Richstone. The Centers of Early-Type Galaxies with HST. IV. Central Parameter Relations. Astron. J., 114:1771, November 1997.

[44] M. C. Begelman, R. D. Blandford, and M. J. Rees. Massive black hole binaries in active galactic nuclei. Nature, 287:307-309, September 1980.

[45] D. Merritt. Mass Deficits, Stalling Radii, and the Merger Histories of Elliptical Galaxies. Astrophys. J., 648:976-986, September 2006.

[46] J. Kormendy and R. Bender. Correlations between Supermassive Black Holes, Velocity Dispersions, and Mass Deficits in Elliptical Galaxies with Cores. Astrophys. J. Lett., 691:L142-L146, February 2009.

[47] C. Zier. Merging of a massive binary due to ejection of bound stars. Mon. Not. Roy. Astron. Soc., 371:L36-L40, September 2006.

[48] C. Zier. Merging of a massive binary due to ejection of bound stars - II. Mon. Not. Roy. Astron. Soc. , 378:1309-1327, July 2007.

[49] A. Sesana, F. Haardt, and P. Madau. Interaction of Massive Black Hole Binaries with Their Stellar Environment. III. Scattering of Bound Stars. Astrophys. J., 686:432-447, October 2008.

[50] H. Baumgardt, A. Gualandris, and S. Portegies Zwart. Ejection of hypervelocity stars from the Galactic Centre by intermediate-mass black holes. Mon. Not. Roy. Astron. Soc. , 372:174182, October 2006.

[51] S. F. Portegies Zwart, H. Baumgardt, S. L. W. McMillan, J. Makino, P. Hut, and T. Ebisuzaki. The Ecology of Star Clusters and Intermediate-Mass Black Holes in the Galactic Bulge. Astrophys. J., 641:319-326, April 2006.

[52] T. Matsubayashi, J. Makino, and T. Ebisuzaki. Orbital Evolution of an IMBH in the Galactic Nucleus with a Massive Central Black Hole. Astrophys. J., 656:879-896, February 2007.

[53] U. Löckmann and H. Baumgardt. Tracing intermediate-mass black holes in the Galactic Centre. Mon. Not. Roy. Astron. Soc., 384:323-330, February 2008.

[54] M. Lang, K. Holley-Bockelmann, T. Bogdanovic, P. Amaro-Seoane, A. Sesana, and M. Sinha. Can a Satellite Galaxy Merger Explain the Active Past of the Galactic Center? Mon. Not. Roy. Astron. Soc. , 430:2574, April 2013.

[55] B. M. S. Hansen and M. Milosavljević. The Need for a Second Black Hole at the Galactic Center. Astrophys. J. Lett., 593:L77-L80, August 2003.

[56] Q. Yu and S. Tremaine. Ejection of Hypervelocity Stars by the (Binary) Black Hole in the Galactic Center. Astrophys. J., 599:1129-1138, December 2003.

[57] S. Trippe, S. Gillessen, O. E. Gerhard, H. Bartko, T. K. Fritz, H. L. Maness, F. Eisenhauer, F. Martins, T. Ott, K. Dodds-Eden, and R. Genzel. Kinematics of the old stellar population at the Galactic centre. Astron. \& Astrophys., 492:419-439, December 2008.

[58] A. Gualandris and D. Merritt. Perturbations of Intermediate-mass Black Holes on Stellar Orbits in the Galactic Center. Astrophys. J., 705:361-371, November 2009.

[59] X. Chen and F. K. Liu. Is There an Intermediate Massive Black Hole in the Galactic Center: Imprints on the Stellar Tidal-disruption Rate. Astrophys. J., 762:95, January 2013.

[60] R. Capuzzo-Dolcetta. The Evolution of the Globular Cluster System in a Triaxial Galaxy: Can 
a Galactic Nucleus Form by Globular Cluster Capture? Astrophys. J., 415:616, October 1993.

[61] R. Capuzzo-Dolcetta and P. Miocchi. Merging of Globular Clusters in Inner Galactic Regions. II. Nuclear Star Cluster Formation. Astrophys. J., 681:1136-1147, July 2008.

[62] F. Antonini. Origin and Growth of Nuclear Star Clusters around Massive Black Holes. Astrophys. J., 763:62, January 2013.

[63] F. Antonini, R. Capuzzo-Dolcetta, A. Mastrobuono-Battisti, and D. Merritt. Dissipationless Formation and Evolution of the Milky Way Nuclear Star Cluster. Astrophys. J., 750:111, May 2012.

[64] F. Antonini. On the distribution of stellar remnants around massive black holes: slow mass segregation, star cluster inspirals and correlated orbits. ArXiv e-prints, February 2014.

[65] H. B. Perets and A. Mastrobuono-Battisti. Age and Mass Segregation of Multiple Stellar Populations in Galactic Nuclei and their Observational Signatures. Astrophys. J. Lett., 784:L44, April 2014.

[66] E. E. Becklin and G. Neugebauer. Infrared Observations of the Galactic Center. Astrophys. J., 151:145, January 1968.

[67] R. Schödel, A. Feldmeier, D. Kunneriath, S. Stolovy, N. Neumayer, P. Amaro-Seoane, and S. Nishiyama. Surface Brightness Profile of the Milky Way's Nuclear Star Cluster. ArXiv e-prints, March 2014.

[68] A. Feldmeier, N. Neumayer, A. Seth, R. Schödel, N. Lützgendorf, P. T. de Zeeuw, M. KisslerPatig, S. Nishiyama, and C. J. Walcher. Large scale kinematics and dynamical modelling of the Milky Way nuclear star cluster. ArXiv e-prints, June 2014.

[69] M. B. Davies, R. Blackwell, V. C. Bailey, and S. Sigurdsson. The destructive effects of binary encounters on red giants in the Galactic Centre. Mon. Not. Roy. Astron. Soc. , 301:745-753, December 1998.

[70] T. Alexander. The Distribution of Stars near the Supermassive Black Hole in the Galactic Center. Astrophys. J., 527:835-850, December 1999.

[71] V. C. Bailey and M. B. Davies. Red giant collisions in the Galactic Centre. Mon. Not. Roy. Astron. Soc., 308:257-270, September 1999.

[72] J. E. Dale, M. B. Davies, R. P. Church, and M. Freitag. Red giant stellar collisions in the Galactic Centre. Mon. Not. Roy. Astron. Soc., 393:1016-1033, March 2009.

[73] P. Amaro-Seoane and X. Chen. The Fragmenting Past of the Disk at the Galactic Center: The Culprit for the Missing Red Giants. Astrophys. J. Lett., 781:L18, January 2014.

[74] Y. Levin and A. M. Beloborodov. Stellar Disk in the Galactic Center: A Remnant of a Dense Accretion Disk? Astrophys. J. Lett., 590:L33-L36, June 2003.

[75] S. Nayakshin and J. Cuadra. A self-gravitating accretion disk in Sgr A* a few million years ago: Is Sgr A* a failed quasar? Astron. \& A Astrophys., 437:437-445, July 2005.

[76] Y. Levin. Starbursts near supermassive black holes: young stars in the Galactic Centre, and gravitational waves in LISA band. Mon. Not. Roy. Astron. Soc. , 374:515-524, January 2007.

[77] P. J. Armitage, W. H. Zurek, and M. B. Davies. Red Giant-Disk Encounters: Food for Quasars? Astrophys. J., 470:237, October 1996.

[78] H. Zinnecker and H. W. Yorke. Toward Understanding Massive Star Formation. Annu. Rev. Astron. Astrophys., 45:481-563, September 2007.

[79] J. Goodman and J. C. Tan. Supermassive Stars in Quasar Disks. Astrophys. J., 608:108-118, June 2004.

[80] S. Nayakshin. Massive stars in subparsec rings around galactic centres. Mon. Not. Roy. Astron. Soc. , 372:143-150, October 2006.

[81] I. A. Bonnell and W. K. M. Rice. Star Formation Around Supermassive Black Holes. Science, 321:1060-, August 2008.

[82] A. Hobbs and S. Nayakshin. Simulations of the formation of stellar discs in the Galactic Centre via cloud-cloud collisions. Mon. Not. Roy. Astron. Soc., 394:191-206, March 2009.

[83] C. Alig, A. Burkert, P. H. Johansson, and M. Schartmann. Simulations of direct collisions of gas clouds with the central black hole. Mon. Not. Roy. Astron. Soc., 412:469-486, March 2011.

[84] M. Mapelli, T. Hayfield, L. Mayer, and J. Wadsley. In Situ Formation of SgrA* Stars Via Disk Fragmentation: Parent Cloud Properties and Thermodynamics. Astrophys. J., 749:168, April 2012.

[85] H. Bartko, F. Martins, S. Trippe, T. K. Fritz, R. Genzel, T. Ott, F. Eisenhauer, S. Gillessen, T. Paumard, T. Alexander, K. Dodds-Eden, O. Gerhard, Y. Levin, L. Mascetti, S. Nayakshin, H. B. Perets, G. Perrin, O. Pfuhl, M. J. Reid, D. Rouan, M. Zilka, and 
A. Sternberg. An Extremely Top-Heavy Initial Mass Function in the Galactic Center Stellar Disks. Astrophys. J., 708:834-840, January 2010.

[86] J. R. Lu, T. Do, A. M. Ghez, M. R. Morris, S. Yelda, and K. Matthews. Stellar Populations in the Central 0.5 pc of the Galaxy. II. The Initial Mass Function. Astrophys. J., 764:155, February 2013.

[87] S. Nayakshin and R. Sunyaev. The 'missing' young stellar objects in the central parsec of the Galaxy: evidence for star formation in a massive accretion disc and a top-heavy initial mass function. Mon. Not. Roy. Astron. Soc., 364:L23-L27, November 2005.

[88] S. Nayakshin, W. Dehnen, J. Cuadra, and R. Genzel. Weighing the young stellar discs around Sgr A*. Mon. Not. Roy. Astron. Soc., 366:1410-1414, March 2006.

[89] R. D. Alexander, M. C. Begelman, and P. J. Armitage. Constraints on the Stellar Mass Function from Stellar Dynamics at the Galactic Center. Astrophys. J., 654:907-914, January 2007.

[90] M. B. Davies and A. King. The Stars of the Galactic Center. Astrophys. J. Lett., 624:L25-L27, May 2005.

[91] J. G. Hills. Hyper-velocity and tidal stars from binaries disrupted by a massive Galactic black hole. Nature, 331:687-689, February 1988.

[92] J. G. Hills. Computer simulations of encounters between massive black holes and binaries. Astron. J., 102:704-715, August 1991.

[93] A. Gould and A. C. Quillen. Sagittarius A* Companion S0-2: A Probe of Very High Mass Star Formation. Astrophys. J., 592:935-940, August 2003.

[94] I. Ginsburg and A. Loeb. The fate of former companions to hypervelocity stars originating at the Galactic Centre. Mon. Not. Roy. Astron. Soc., 368:221-225, May 2006.

[95] H. B. Perets, C. Hopman, and T. Alexander. Massive Perturber-driven Interactions between Stars and a Massive Black Hole. Astrophys. J., 656:709-720, February 2007.

[96] H. B. Perets and A. Gualandris. Dynamical Constraints on the Origin of the Young B-stars in the Galactic Center. Astrophys. J., 719:220-228, August 2010.

[97] S. Nayakshin, J. Cuadra, and V. Springel. Simulations of star formation in a gaseous disc around Sgr A* - a failed active galactic nucleus. Mon. Not. Roy. Astron. Soc., 379:21-33, July 2007.

[98] J. Binney and S. Tremaine. Galactic Dynamics: Second Edition. Princeton University Press, 2008.

[99] K. P. Rauch and S. Tremaine. Resonant relaxation in stellar systems. New Astron., 1:149-170, October 1996.

[100] K. P. Rauch and B. Ingalls. Resonant tidal disruption in galactic nuclei. Mon. Not. Roy. Astron. Soc. , 299:1231-1241, October 1998.

[101] H. B. Perets, A. Gualandris, G. Kupi, D. Merritt, and T. Alexander. Dynamical Evolution of the Young Stars in the Galactic Center: N-body Simulations of the S-Stars. Astrophys. J., 702:884-889, September 2009.

[102] A.-M. Madigan, C. Hopman, and Y. Levin. Secular Stellar Dynamics near a Massive Black Hole. Astrophys. J., 738:99, September 2011.

[103] F. Antonini and D. Merritt. Relativity and the Evolution of the Galactic Center S-star orbits. Astrophys. J. Lett., 763:L10, January 2013.

[104] F. Zhang, Y. Lu, and Q. Yu. The Galactic Center S-stars and the Hypervelocity Stars in the Galactic Halo: Two Faces of the Tidal Breakup of Stellar Binaries by the Central Massive Black Hole? Astrophys. J., 768:153, May 2013.

[105] A. S. Hamers, S. F. Portegies Zwart, and D. Merritt. Relativistic dynamics of stars near a supermassive black hole. ArXiv e-prints, June 2014.

[106] F. Eisenhauer, R. Schödel, R. Genzel, T. Ott, M. Tecza, R. Abuter, A. Eckart, and T. Alexander. A Geometric Determination of the Distance to the Galactic Center. Astrophys. J. Lett., 597:L121-L124, November 2003.

[107] F. Martins, S. Gillessen, F. Eisenhauer, R. Genzel, T. Ott, and S. Trippe. On the Nature of the Fast-Moving Star S2 in the Galactic Center. Astrophys. J. Lett., 672:L119-L122, January 2008.

[108] S. Yelda, A. M. Ghez, J. R. Lu, T. Do, L. Meyer, M. R. Morris, and K. Matthews. Properties of the Remnant Clockwise Disk of Young Stars in the Galactic Center. Astrophys. J., 783:131, March 2014.

[109] D. Merritt, A. Gualandris, and S. Mikkola. Explaining the Orbits of the Galactic Center S-Stars. Astrophys. J. Lett., 693:L35-L38, March 2009.

[110] I. Bartos, Z. Haiman, B. Kocsis, and S. Márka. Gas Cloud G2 Can Illuminate the Black Hole Population Near the Galactic Center. Physical Review Letters, 110(22):221102, May 2013.

[111] X. Chen and P. Amaro-Seoane. A Rapidly Evolving Region in the Galactic Center: Why 
S-stars Thermalize and More Massive Stars are Missing. Astrophys. J. Lett., 786:L14, May 2014.

[112] Y. Kozai. Secular perturbations of asteroids with high inclination and eccentricity. Astron. J., 67:591, November 1962.

[113] M. L. Lidov. The evolution of orbits of artificial satellites of planets under the action of gravitational perturbations of external bodies. Planetary and Space Sci., 9:719-759, October 1962.

[114] S. Naoz, W. M. Farr, Y. Lithwick, F. A. Rasio, and J. Teyssandier. Secular dynamics in hierarchical three-body systems. Mon. Not. Roy. Astron. Soc. , 431:2155-2171, May 2013.

[115] L. Šubr and V. Karas. On highly eccentric stellar trajectories interacting with a self-gravitating disc in Sgr A*. Astron. \& Astrophys., 433:405-413, April 2005.

[116] U. Löckmann, H. Baumgardt, and P. Kroupa. Origin of the S Stars in the Galactic Center. Astrophys. J. Lett., 683:L151-L154, August 2008.

[117] U. Löckmann, H. Baumgardt, and P. Kroupa. Influence of a stellar cusp on the dynamics of young stellar discs and the origin of the S-stars in the Galactic Centre. Mon. Not. Roy. Astron. Soc. , 398:429-437, September 2009.

[118] P. Chang. The effectiveness of the Kozai mechanism in the Galactic Centre. Mon. Not. Roy. Astron. Soc. , 393:224-228, February 2009.

[119] M. Wardle and F. Yusef-Zadeh. On the Formation of Compact Stellar Disks around Sagittarius A*. Astrophys. J. Lett., 683:L37-L40, August 2008.

[120] T. Paumard, R. Genzel, F. Martins, S. Nayakshin, A. M. Beloborodov, Y. Levin, S. Trippe, F. Eisenhauer, T. Ott, S. Gillessen, R. Abuter, J. Cuadra, T. Alexander, and A. Sternberg. The Two Young Star Disks in the Central Parsec of the Galaxy: Properties, Dynamics, and Formation. Astrophys. J., 643:1011-1035, June 2006.

[121] M. Wardle and F. Yusef-Zadeh. On the Origin of the Central 1" Hole in the Stellar Disk of Sgr A* and the Fermi Gamma-Ray Bubbles. Astrophys. J. Lett., 787:L14, May 2014.

[122] R. D. Alexander, S. L. Smedley, S. Nayakshin, and A. R. King. Galactic Centre star formation: the case of the missing gas disc. Mon. Not. Roy. Astron. Soc. , 419:1970-1976, January 2012.

[123] C. Hopman and T. Alexander. Resonant Relaxation near a Massive Black Hole: The Stellar Distribution and Gravitational Wave Sources. Astrophys. J., 645:1152-1163, July 2006.

[124] B. Kocsis and S. Tremaine. Resonant relaxation and the warp of the stellar disc in the Galactic Centre. Mon. Not. Roy. Astron. Soc., 412:187-207, March 2011.

[125] B. Paczyńsky and P. J. Wiita. Thick accretion disks and supercritical luminosities. Astron. E Astrophys., 88:23-31, August 1980.

[126] X. Chen, F. K. Liu, and J. Magorrian. Tidal Disruption of Stellar Objects by Hard Supermassive Black Hole Binaries. Astrophys. J., 676:54-69, March 2008.

[127] X. Chen, A. Sesana, P. Madau, and F. K. Liu. Tidal Stellar Disruptions by Massive Black Hole Pairs. II. Decaying Binaries. Astrophys. J., 729:13, March 2011.

[128] C. Wegg and J. Nate Bode. Multiple Tidal Disruptions as an Indicator of Binary Supermassive Black Hole Systems. Astrophys. J. Lett., 738:L8, September 2011.

[129] P. B. Ivanov, A. G. Polnarev, and P. Saha. The tidal disruption rate in dense galactic cusps containing a supermassive binary black hole. Mon. Not. Roy. Astron. Soc. , 358:1361-1378, April 2005.

[130] B. Kocsis and S. Tremaine. A numerical study of vector resonant relaxation. ArXiv e-prints, June 2014.

[131] A. Tanner, D. F. Figer, F. Najarro, R. P. Kudritzki, D. Gilmore, M. Morris, E. E. Becklin, I. S. McLean, A. M. Gilbert, J. R. Graham, J. E. Larkin, N. A. Levenson, and H. I. Teplitz. High Spectral Resolution Observations of the Massive Stars in the Galactic Center. Astrophys. J., 641:891-904, April 2006.

[132] J. R. Lu, A. M. Ghez, S. D. Hornstein, M. R. Morris, E. E. Becklin, and K. Matthews. A Disk of Young Stars at the Galactic Center as Determined by Individual Stellar Orbits. Astrophys. J., 690:1463-1487, January 2009.

[133] H. Bartko, F. Martins, T. K. Fritz, R. Genzel, Y. Levin, H. B. Perets, T. Paumard, S. Nayakshin, O. Gerhard, T. Alexander, K. Dodds-Eden, F. Eisenhauer, S. Gillessen, L. Mascetti, T. Ott, G. Perrin, O. Pfuhl, M. J. Reid, D. Rouan, A. Sternberg, and S. Trippe. Evidence for Warped Disks of Young Stars in the Galactic Center. Astrophys. J., 697:17411763, June 2009.

[134] E. Griv. Origin of the Galactic Center S-Stars: Gravitational Torques from Lin-Shu-Type Spiral Density Waves. Astrophys. J., 709:597-604, February 2010.

[135] A.-M. Madigan, Y. Levin, and C. Hopman. A New Secular Instability of Eccentric Stellar Disks 
around Supermassive Black Holes, with Application to the Galactic Center. Astrophys. J. Lett., 697:L44-L48, May 2009.

[136] A. Gualandris, M. Mapelli, and H. B. Perets. Eccentric disc instability in stellar discs formed from inspiralling gas clouds in the Galactic Centre. Mon. Not. Roy. Astron. Soc. , 427:17931799, December 2012.

[137] L. Subr and J. Haas. Two-body Relaxation Driven Evolution of the Young Stellar Disk in the Galactic Center. Astrophys. J., 786:121, May 2014.

[138] J. Sanchez-Bermudez, R. Schödel, A. Alberdi, K. Muzic, C. A. Hummel, and J.-U. Pott. The properties of bow-shock sources at the Galactic Center. ArXiv e-prints, May 2014.

[139] T. Ott, A. Eckart, and R. Genzel. Variable and Embedded Stars in the Galactic Center. Astrophys. J., 523:248-264, September 1999.

[140] F. Martins, S. Trippe, T. Paumard, T. Ott, R. Genzel, G. Rauw, F. Eisenhauer, S. Gillessen, H. Maness, and R. Abuter. GCIRS 16SW: A Massive Eclipsing Binary in the Galactic Center. Astrophys. J. Lett., 649:L103-L106, October 2006.

[141] M. Rafelski, A. M. Ghez, S. D. Hornstein, J. R. Lu, and M. Morris. Photometric Stellar Variability in the Galactic Center. Astrophys. J., 659:1241-1256, April 2007.

[142] O. Pfuhl, T. Alexander, S. Gillessen, F. Martins, R. Genzel, F. Eisenhauer, T. K. Fritz, and T. Ott. Massive Binaries in the Vicinity of Sgr A*. Astrophys. J., 782:101, February 2014.

[143] J. G. Hills. Possible power source of Seyfert galaxies and QSOs. Nature, 254:295-298, March 1975.

[144] M. J. Rees. Tidal disruption of stars by black holes of 10 to the 6 th-10 to the 8 th solar masses in nearby galaxies. Nature, 333:523-528, June 1988.

[145] L. Meyer, A. M. Ghez, R. Schödel, S. Yelda, A. Boehle, J. R. Lu, T. Do, M. R. Morris, E. E. Becklin, and K. Matthews. The Shortest-Known-Period Star Orbiting Our Galaxy's Supermassive Black Hole. Science, 338:84-, October 2012.

[146] K. S. Cheng, D. O. Chernyshov, and V. A. Dogiel. Annihilation Emission from the Galactic Black Hole. Astrophys. J., 645:1138-1151, July 2006.

[147] K. S. Cheng, D. O. Chernyshov, and V. A. Dogiel. Diffuse gamma-ray emission from the Galactic center - a multiple energy injection model. Astron. ES Astrophys., 473:351-356, October 2007.

[148] G. Ponti, R. Terrier, A. Goldwurm, G. Belanger, and G. Trap. Discovery of a Superluminal Fe K Echo at the Galactic Center: The Glorious Past of Sgr A* Preserved by Molecular Clouds. Astrophys. J., 714:732-747, May 2010.

[149] R. Capelli, R. S. Warwick, D. Porquet, S. Gillessen, and P. Predehl. The X-ray lightcurve of Sagittarius A* over the past 150 years inferred from Fe-K $\alpha$ line reverberation in Galactic centre molecular clouds. Astron. ES Astrophys., 545:A35, September 2012.

[150] M. Su, T. R. Slatyer, and D. P. Finkbeiner. Giant Gamma-ray Bubbles from Fermi-LAT: Active Galactic Nucleus Activity or Bipolar Galactic Wind? Astrophys. J., 724:1044-1082, December 2010.

[151] M. Su and D. P. Finkbeiner. Evidence for Gamma-Ray Jets in the Milky Way. Astrophys. J., 753:61, July 2012

[152] F. Yusef-Zadeh, R. Arendt, H. Bushouse, W. Cotton, D. Haggard, M. W. Pound, D. A. Roberts, M. Royster, and M. Wardle. A 3 pc Scale Jet-driven Outflow from Sgr A*. Astrophys. J. Lett., 758:L11, October 2012.

[153] J. Bland-Hawthorn, P. R. Maloney, R. S. Sutherland, and G. J. Madsen. Fossil Imprint of a Powerful Flare at the Galactic Center along the Magellanic Stream. Astrophys. J., 778:58, November 2013.

[154] P. F. Hopkins and L. Hernquist. Fueling Low-Level AGN Activity through Stochastic Accretion of Cold Gas. Astrophys. J. Sup. Ser., 166:1-36, September 2006.

[155] M. Volonteri, M. Sikora, J.-P. Lasota, and A. Merloni. The Evolution of Active Galactic Nuclei and their Spins. Astrophys. J., 775:94, October 2013.

[156] A. R. King, S. H. Lubow, G. I. Ogilvie, and J. E. Pringle. Aligning spinning black holes and accretion discs. Mon. Not. Roy. Astron. Soc. , 363:49-56, October 2005.

[157] G. Lodato and J. E. Pringle. The evolution of misaligned accretion discs and spinning black holes. Mon. Not. Roy. Astron. Soc., 368:1196-1208, May 2006.

[158] M. Volonteri, M. Sikora, and J.-P. Lasota. Black Hole Spin and Galactic Morphology. Astrophys. J., 667:704-713, October 2007.

[159] M. Dotti, M. Colpi, S. Pallini, A. Perego, and M. Volonteri. On the Orientation and Magnitude of the Black Hole Spin in Galactic Nuclei. Astrophys. J., 762:68, January 2013. 